\title{
BOUNDED SECTIONS ON A RIEMANN SURFACE
}

\author{
WALTER PRANGER
}

\begin{abstract}
Let $X$ denote a hyperbolic Riemann surface, $\zeta$ a unitary line bundle, and $H^{\infty}(\zeta)$ the Banach space of bounded holomorphic sections of $\zeta$. If, for a given point $\xi$ in $X$, the norm of the evaluation functional on $H^{\infty}(\zeta)$ varies continuously with the bundle $\zeta$, then it is shown that the space of bounded holomorphic sections is dense in the space of holomorphic sections for every unitary line bundle.
\end{abstract}

The results of this note are related to the work of Harold Widom in [1] and [2]. They center around the following data-a connected hyperbolic Riemann surface $X$, and equivalence class of unitary line bundles $\zeta$ on $X$, and the space $B(X, \zeta)$ of bounded holomorphic sections of $\zeta$. The function $m_{\infty}$ is defined as $m_{\infty}(X, \zeta, z)=\sup |f(z)|$ where $f \in B(X, \zeta)$ and $|f| \leqslant 1$. The function $m_{\infty}$ is a map from $G^{*}$, the group of equivalence classes of unitary line bundles, to the interval $0 \leqslant x \leqslant 1$. The group $G^{*}$ is the character group of the first homology group $H_{1}(X, Z)$ and as such is given the weak topology. In [2] there are several interesting consequences of the assumption that $m_{\infty}$ be continuous. The surfaces for which this is true have properties similar to the disk as concerns bounded holomorphic functions. One of these properties is that such a surface $X$ is $B$-convex, i.e., the space $B(X)$ of bounded holomorphic functions is dense in the space $H(X)$ of holomorphic functions. This is established below.

THEOREM. If the map $m_{\infty}$ is continuous, then

(a) $X$ is $B$-convex,

(b) $B(X)$ is dense in $H_{p}(X)$ for $p>0$ and in $N^{+}(X)$, and

(c) $X$ is regular for potential theory.

The space $N^{+}(X)$ is the Smirnov class for $X$ and $N(X)$ is the Nevanlinna class. It should be noted that in [2] it is asserted that if $m_{\infty}$ is continuous, then $B(X)$ is dense in $N(X)$. This cannot be true in general. The argument there proves the assertion that $B(X)$ is dense in $N^{+}(X)$. Even when the surface is a disk, $B$ is not dense in $N$ since $N^{+}$, which contains $B$, is a closed proper subspace of $N$ (see [3] for the definitions and facts; especially p. 919).

The proof of (b) appears in [2]. The proof of (c) follows from (a) and recent work of Hasumi in the following way. Results of [4, p. 276] show that

Presented to the Society, August 17, 1977; received by the editors March 1, 1977 and, in revised form, July $22,1977$.

AMS (MOS) subject classifications (1970). Primary 46J15, $30 \mathrm{~A} 98$.

(c) American Mathematical Society 1978 
whenever every $B(X, \zeta)$ has nonzero members (which is the case when $m_{\infty}$ is continuous), then $X$ is a surface $Y$ less a discrete set and $Y$ is regular for potential theory. If (a) holds, then clearly $X=Y$.

Now I prove (a). A necessary condition for the continuity of $m_{\infty}$ is that every unitary line bundle have nonzero bounded holomorphic sections. This in turn means that $B(X)$ separates points and provides local coordinates. To show the $B$-convexity of $X$ I use the properties of algebras of holomorphic functions on a Riemann surface as developed in [5], [6] and [7]. The algebra to which these results will be applied is $l(X)$, the closure in $H(X)$ of the bounded holomorphic functions. Using [6, Theorem 2, p. 508] there is a surface $Y$ and a morphism $\phi$ from $X$ to $Y$ and an algebra $A^{\prime}$ on $Y$ and a map $\tau$ from $l(X)$ to $A^{\prime}$ which is bijective and $(\tau(f)) \circ \phi=f$. Because $l(X)$ separates, $\phi$ is injective and I may suppose $X \subseteq Y, \phi$ is inclusion and $\tau$ is restriction to $X$. Condition (i) of [6, Theorem 2, p. 508] implies that for every compact set $k \subset Y$ there is a compact set $k_{0}$ in $X$ for which

$$
k \subset\left\{z|| f(z) \mid \leqslant\|f\|_{k_{0}} \forall f \in A^{\prime}\right\} .
$$

If $f \in B(X)$, then $f$ extends to a member of $A^{\prime}$; hence on $k,|f(z)| \leqslant\|f\|_{k_{0}} \leqslant$ $\|f\|_{X}$. Since $k$ is an arbitrary compact set of $Y, f \in B(Y)$ and the norm of $f$ on $Y$ is the same as its norm on $X$. So $B(X)$ is $B(Y)$ restricted to $X$. Also I claim that $A^{\prime}$ is $l(Y)$. Since $B(X)$ and $B(Y)$ are identical $l(Y) \subseteq A^{\prime}$ and $A^{\prime}$ is the set of $F \in H(Y),\left.F\right|_{X} \in l(X)$. So $F$ is the uniform limit on compact subsets of $X$ of members of $B(X)$. Condition (1) and the fact that $B(X)=$ $\left.B(Y)\right|_{X}$ show that convergence on $X$ extends to $Y$. So $A^{\prime}=l(Y)$. Using (iv) of [6, Theorem 2, p. 508] for every compact $k$,

$$
k\left(A^{\prime}\right)=\left\{z|z \in Y,| f(z) \mid \leqslant\|f\|_{k} \forall f \in A^{\prime}\right\}
$$

is compact and consists of $k$ union with those components of the complement of $k$ which are relatively compact. Since $B(Y)$ closure is $l(Y)=A^{\prime}, \hat{k}\left(A^{\prime}\right)=$ $\hat{k}(B(Y))$.

Now I show that $X=Y$. In what follows the relation between sections of unitary line bundles and multifunctions which are holomorphic and whose absolute values are functions will be exploited as in [1]. Two multifunctions whose absolute values are functions give rise to the same section provided they have identical moduli. Let $a$ belong to the closure of $X$ but not to $X$ and let $0 \leqslant t \leqslant 1$. There is a holomorphic multifunction $f_{t}$ on $Y-\{a\}$ such that $\left|f_{t}\right|=\exp \left(-t \cdot g_{a}\right)$; and $f_{t}$ corresponds to a section of some $\xi_{t}$ on $Y-\{a\}$. I can restrict $\xi_{t}$ to $X$ and choose an $h \in B\left(X, \bar{\xi}_{t}\right)$ so $|h| \leqslant 1$ and $h$ is not identically zero. Then $h f_{t}=F \in B(X)$ so extends to $B(Y)$. On $Y,|F|=$ $\exp (-u-p)$, where $u$ is a positive harmonic function and $p$ is a discrete Green potential; because $|F| \leqslant 1$ on $X$ means $|h|=\exp \left(-u-p+\operatorname{tg}_{a}\right) \leqslant 1$ on $Y-\{a\}$ so $u+p-\operatorname{tg}_{a} \geqslant 0$ on $Y-\{a\}$ or $u+p \geqslant \operatorname{tg}_{a}$ and this last inequality holds on $Y$. This can be the case only if $p=g_{a}+q$ where $q$ is a discrete Green potential. Therefore

$$
|h|=\exp \left(-u-g_{a}-q+t g_{a}\right) \leqslant \exp \left(-(1-t) g_{a}\right)
$$


Hence,

$$
m_{\infty}\left(X, \bar{\xi}_{t}, z\right) \leqslant \exp \left(-(1-t) g_{a}(z)\right) .
$$

As $t \rightarrow 0, f_{t} \rightarrow 1$ so $\xi_{t} \rightarrow i_{d}$ and $\bar{\xi}_{t} \rightarrow i_{d}$. The assumed continuity of $m_{\infty}$ on $X$ means $m_{\infty}\left(X, \bar{\xi}_{t}, z\right)$ increases to 1 . But from $(1), 1 \leqslant \exp \left(-g_{a}(z)\right)<1$, a contradiction. Thus $X=Y$.

Now it follows that for every compact $k \subset X, \hat{k}_{\infty}=\left\{z|| f(z) \mid<\|f\|_{k} \forall\right.$ $f \in B(X)\}$ is compact and equals $\hat{k}=\left\{z|| f(z) \mid<\|f\|_{k} \forall f \in H(X)\right\}$. Moreover, $X$ can be exhausted by compact sets $k$ for which $k=\hat{k}=\hat{k}_{\infty}[8$, p. 240]. The result of Bishop [5, Corollary 2, p. 48] can be applied to conclude that on any such compact set $k=\hat{k}=\hat{k}_{\infty}$ every function continuous on $k$ and holomorphic on its interior is the uniform limit of members of $l(X)$, hence of members of $B(X)$. Thus $X$ is $B$-convex.

Now I can use the result that $X$ is $B$-convex to prove the stronger statement that $B(X, \zeta)$ is dense in $H(X, \zeta)$ for every unitary line bundle $\zeta$.

TheOrem. If $m_{\infty}$ is continuous, then $B(X, \zeta)$ is dense in $H(X, \zeta)$ for every $\zeta$.

The first step in the proof is to show that for every compact set $K$ and every unitary line bundle $\zeta$ there is a section $f \in B(X, \zeta)$ which has no zeros on $K$. Let $A=\{\zeta \mid$ there is an $f \in B(X, \zeta)$ with no zeros on $K\} . A$ is a subset of $G^{*}$ and has these properties- $i_{d} \in A$; if $\xi, \eta \in A$, then $\xi \eta \in A$; and $A$ contains an open set about $i_{d}$. Only the last property needs verification. I suppose that each neighborhood of $i_{d}$ has a $\xi$ with the property that every $f \in B(X, \xi)$ has a zero on $K$. In particular, if $f$ is an extremal section at $a$ for $\xi$ normalized so $f(a)=m_{\infty}(X, \xi, a)$, then $f$ has a zero on $K$ for certain $\xi$ close to $i_{d}$. As a consequence there is a sequence of unitary line bundles $\left\{\xi_{j}\right\}$ converging to $i_{d}$ and a corresponding sequence of points $\left\{z_{j}\right\}$ in $K$ such that the corresponding extremal $f_{j}$ for $\xi_{j}$ at $a$ satisfies $\left|f_{j}\left(z_{j}\right)\right|=0$. I may assume that the sequence $\left\{z_{j}\right\}$ converges to some $b \in K$ and at the same time, that $\left\{f_{j}\right\}$ converges on $K$ uniformly to a function $f \in B(X)$. By the assumed continuity of $m_{\infty}, m_{\infty}\left(\xi_{j}, a\right)=f_{j}(a)$ converges to 1 . Since $|f| \leqslant 1$ and $f(a)=$ 1 , I must have $f \equiv 1$. Now $b$ is a limit of $\left\{z_{j}\right\}$ so $|f(b)|=\lim \left|f_{j}\left(z_{j}\right)\right|=0$ which is a contradiction. Since $G^{*}$ is both compact and connected it follows that $G^{*}=A$.

To conclude the proof of the theorem let $K$ denote a compact set such that $K=\hat{K}_{\infty}=\hat{K}$ (the conclusion of Theorem 1 and the fact that $X$ is a Stein manifold means $X$ can be exhausted by such sets). Let $f \in H(X, \xi)$ and let $b \in B(X, \xi)$ so $b$ has no zeros on a neighborhood of $K$. Let $\varepsilon>0$ be given. Since $K=\hat{K}$ and since $X$ is holomorph-convex and $f / b$ is holomorphic on a neighborhood of $K$, there is an $F \in H(X)$ for which $|F-f / b|<\varepsilon\|b\|^{-1}$ on $K$. As $X$ is $B$-convex I may take it that $F \in B(X)$. Then $|f-b F|<\varepsilon$ on $K$. Since $b F \in B(X, \xi)$, the assertion of the theorem follows.

Referring to [9] one can remove from the punctured disk $0<|z|<1$ a sequence of closed disks so that the resulting surface $X$ has the property that 
the origin is not a regular point for potential theory. On the other hand $X$ is $B$-convex. This is easily seen to be the case since $B(X)$ separates, provides local coordinates, and $\hat{k}_{\infty}$ is compact for every compact $k$. It follows that $X$ is $B$-convex but $m_{\infty}$ is not continuous.

One can interpret $m_{\infty}$ in terms of linear functionals in the following way. If $F$ is a section of $\bar{\zeta}$ such that $|F(\xi)|=1$, then the map which sends $f$ onto $(F f)(\xi)=L_{\zeta}(f)$ is a continuous linear functional on $H^{\infty}(X, \zeta)(B(X, \zeta)$ with the norm topology). Then $m_{\infty}(\zeta)=\left\|L_{\zeta}\right\|$; so the continuity of $m_{\infty}$ means that the norm varies continuously with $\zeta$, the unitary line bundle.

\section{REFERENCES}

1. Harold Widom, $H_{p}$ sections of vector bundles over Riemann surfaces, Ann. of Math. (2) 94 (1971), 304-324.

2. The maximum principle for multivalued analytic functions, Acta Math. 126 (1971), 63-82.

3. Joel H. Shapiro and Allen L. Shields, Unusual topological properties of the Nevanlinna class, Amer. J. Math. 97 (1976), 915-956.

4. Morisuke Hasumi, Invariant subspaces on open Riemann surfaces. II, Ann. Inst. Fourier (Grenoble) 26 (1976), fasc. 2, 273-299.

5. E. Bishop, Subalgebras of functions on a Riemann surface, Pacific J. Math. 8 (1958), 29-50.

6. __ Analyticity in certain Banach algebras, Trans. Amer. Math. Soc. 102 (1962), 507-544.

7. H. L. Royden, Algebras of bounded analytic functions on Riemann surfaces, Acta Math. 114 (1965), 113-142.

8. R. Narasimhan, Analysis on real and complex manifolds, North-Holland, Amsterdam, 1973.

9. L. Zalcman, Bounded analytic functions on domains of infinite connectivity, Trans. Amer. Math. Soc. 144 (1969), 241-269.

Department of Mathematics, DePaul University, Chicago, Illinois 60614 